\title{
Color Filter Array Interpolation Based on Spatial Adaptivity
}

\author{
Dmitriy Paliy $^{a}$, Radu Bilcu ${ }^{b}$, Vladimir Katkovnik ${ }^{a}$, Markku Vehviläinen $^{b}$ \\ ${ }^{a}$ Institute of Signal Processing, Tampere University of Technology, P.O.Box 553, FIN-33101 \\ Tampere, Finland. \\ e-mail: firstname.lastname@tut.fi \\ ${ }^{b}$ Nokia Research Center, Tampere, Finland. \\ e-mail: firstname.lastname@nokia.com
}

\begin{abstract}
Conventional approach in single-chip digital cameras is a use of color filter arrays (CFA) in order to sample different spectral components. Demosaicing algorithms interpolate these data to complete red, green, and blue values for each image pixel, in order to produce an RGB image. In this paper we propose a novel demosaicing algorithm for the Bayer CFA. For the algorithm design we assume that the initial interpolation estimates of color channels contain two additive components: the true values of color intensities and the errors. The errors are considered as an additive noise, and often called as a demosaicing noise, that has to be removed. This noise is not white and strongly depends on the signal. Usually, the intensity of this noise is higher near edges of image details. We use specially designed signal-adaptive filter to remove the interpolation errors. This filter is based on the local polynomial approximation (LPA) and the paradigm of the intersection of confidence intervals (ICI) applied for selection adaptively varying scales (window sizes) of LPA. The LPA-ICI technique is nonlinear and spatially-adaptive with respect to the smoothness and irregularities of the image. The efficiency of the proposed approach is demonstrated by simulation results.
\end{abstract}

Keywords: Bayer pattern, color filter array interpolation, spatially adaptive denoising

\section{INTRODUCTION}

The common approach in single-chip digital cameras is a use of CFA to sample different spectral components like red, green, and blue. The Bayer CFA patented in $1976^{1}$ is widely exploited today (see Fig.1). Demosaicing algorithm interpolates sets of complete red, green, and blue values for each pixel, to make an RGB image. Independent interpolation of color channels usually leads to drastic color distortions. The way how produce effectively a joint color interpolation plays a crucial role for demosaicing.

The modern efficient algorithms exploit two main facts. The first is that for natural images there is a high correlation between the red, green, and blue channels. As a result all three color channels are very likely to have the same texture and edge locations. The second fact is that digital cameras use the CFA in which the luminance (green) channel is sampled at the higher rate than the chrominance (red and blue) channels. Therefore, the green channel is less likely to be aliased, and details are preserved better in the green channel than in the red and blue channels $^{2}$.

\subsection{Correlation models}

There are two basic interplane correlation models in the literature: the color difference rule ${ }^{4,5}$ and the color ratio rule ${ }^{6,7}$.

The first model asserts that intensity differences between red, green, and blue channels are slowly varying, that is the differences between color channels are locally nearly-constant ${ }^{3,4,5,8,9,10,11,12,13,14}$. Thus, they contain low-frequency components only, making the interpolation using the color differences easier ${ }^{10}$.

The second correlation model is based on the assumption that the ratios between colors are constant over some local regions ${ }^{6,7}$. This hypothesis follows from the Lambert's law that if two colors have equal chrominance then the ratios between the intensities of three color components are equal ${ }^{10}$. According to this model, the intensity of each color channel is calculated as a projection of the normal vector to the object surface onto the 


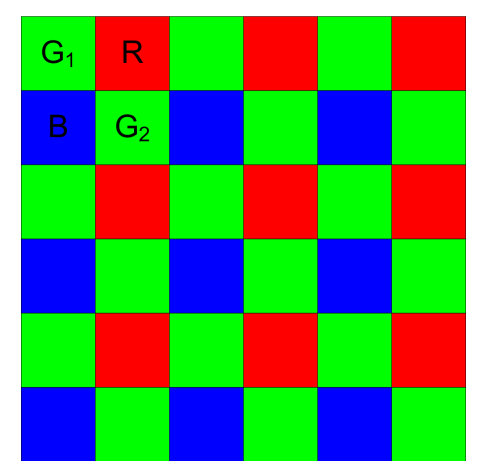

Figure 1. Bayer color filter array.

light source direction, multiplied by the surface albedo. The albedo captures the characteristics of the object's material, and is different for each of the spectral channels. It is assumed that the material, and therefore the albedo, are locally the same within the given object in the image.

The first difference-based correlation model is found to be more efficient than the ratio-based model and, therefore, exploited more often in practice. Moreover, the color-difference rule can be implemented with a lower computational cost and better fits linear interpolation models ${ }^{8}$.

\subsection{Demosaicing methods}

Many demosaicing algorithms $4,5,6,15,16,18,19$ incorporate edge directionality in interpolation. Interpolation along object boundaries is preferable as compared with interpolation across these boundaries for the most image models.

We will classify the demosaicing techniques into two categories: noniterative ${ }^{3,4,5,9,11,12,13,19,20,21,22}$, and iterative $\mathrm{e}^{2,6,7,8,10}$. There are also alternative ways of this classification, for instance proposed in $^{23}$.

Noniterative demosaicing techniques basically rely on the idea of edge-directed interpolation. The exploitation of this sort of intraplane correlation typically is done by estimating local gradients under the main assumption that locally the difference between colors is nearly constant. At each pixel the gradient is estimated, and the color interpolation is carried out directionally, based on this estimated gradient. Directional filtering is the most popular approach for color demosaicing that produces competitive results. The best known directional interpolation scheme is perhaps the method proposed by Hamilton and Adams ${ }^{5}$. The authors use the gradients of blue and red channels as the correction terms to interpolate the green channel. Once the green samples are filled, the red and blue samples are interpolated in analogous way ${ }^{11}$. Similar idea is exploited effectively in ${ }^{4,9,13}$ with the difference in fusing of vertical and horizontal estimates.

In a variety of color demosaicing techniques the gradient estimates analysis plays a central role in reconstructing sharp edges. A new idea in this field has been proposed and effectively used in the papers ${ }^{8,11}$ in 2005 , where the authors filter the differences between the color channels.

Since the color channels of a natural image are highly correlated, the difference signal between the green channel and the red or blue channels constitutes a smoother (low-pass) process. Furthermore, it is observed that this color difference signal is largely uncorrelated to the interpolation errors of the gradient-guided color demosaicing methods, which are band-pass processes. The authors exploit linear minimum mean square-error estimation (LMMSE) for estimating the color difference signals. The LMMSE estimates are obtained in both horizontal and vertical directions, and then fused optimally to remove the demosaicing noise. Finally, the fullresolution three color channels are reconstructed from the LMMSE filtered difference signals ${ }^{11}$.

$\mathrm{In}^{20}$ the method is proposed, where the green channel is used to determine the pattern at a particular pixel, and then a missing red (blue) pixel value is estimated as a weighted average of the neighboring pixels according to this pattern. 
In addition, there are pattern recognition ${ }^{26}$, restoration-based ${ }^{21,27}$, and sampling theory point of view ${ }^{3,12}$ approaches ( $\mathrm{see}^{23}$ for more details).

Methods called template matching have been put forth $\mathrm{in}^{26}$. They estimate edges in the Bayer CFA image and change the interpolation procedure according to edge behavior. This approach works quite well, despite the problem with selection of the threshold for estimation. The algorithm varies with the content of a particular image and can become computationally heavy ${ }^{25}$.

Methods using the regularization theory ${ }^{28}$ or the Bayesian approach ${ }^{29}$ have also been developed. Taubman ${ }^{21}$ proposed a preconditioned efficient approach of Bayesian demosaicing that is used in some digital cameras today.

Alleysson et al. ${ }^{24,25}$ proposed a model showing that luminance and opponent chromatic signals are well localized in the Fourier spectrum. This spatial Fourier-transform information is used to develop a color demosaicing algorithm by selecting appropriate spatial frequencies corresponding to luminance and opponent chromatic signals.

Iterative demosaicing techniques have also been proposed recently ${ }^{2,6,8,10}$. The refinement of green pixels and red/blue pixels are mutually dependent and jointly beneficial to each other. It is natural to introduce an iterative strategy to handle such type of problem ${ }^{8}$.

Kimmel $^{6}$ in 1999 proposed one of the first methods where the iterative approach was introduced and described. According to this method green and red/blue channels are iteratively updated by enforcing the color ratio rule.

Gunturk et. al. proposed in 2002 an approach using filter-bank for decomposition an image to low- and highfrequency subbands ${ }^{2}$ that performs undecimated wavelet transform. This is done in both vertical and horizontal directions. The result is four subbands which contain low- and high-frequency components (LL, LH, HL, HH). Because of the fact that the LH, HL, HH components of different channels are highly correlated they are replaced by the corresponding components of another channel, but at unknown positions only.

$\mathrm{Xin} \mathrm{Li}^{8}$ proposed an iterative color-difference interpolation. This approach successively refines the estimate of missing data by enforcing the color-difference rule at each iteration.

Three inherent problems often associated with demosaicing algorithms that incorporate directional twodimensional interpolation are misguidance color artifacts, interpolation artifacts, and aliasing. The proposed in ${ }^{10}$ demosaicing algorithm, which also adopts the directional interpolation approach, addresses these problems explicitly. In the process of estimating the missing pixel components, the aliasing problem is resolved by applying filterbank techniques to directional interpolation. This interpolation procedure produces two images: horizontally interpolated and vertically interpolated ones. The level of misguidance color artifacts present in these images is compared by a color image homogeneity metric. The misguidance color artifacts are minimized by only keeping the pixels interpolated in the direction with fewer artifacts. The interpolation artifacts are reduced using a nonlinear iterative procedure ${ }^{10}$.

$\mathrm{In}^{30,31}$ authors propose to use directional estimates under assumption that color-difference is constant. These estimates are fused together by means of weighted average which gives some advantages over usual use of just vertical and horizontal estimates. However, the authors also propose the method of postprocessing based on the assumption that the localized color-ratio is constant ${ }^{7}$.

It has been observed that iterative demosaicing techniques are capable often of achieving higher quality in the reconstructed images than noniterative ones at the price of increased computational $\operatorname{cost}^{8}$.

This work was inspired by ${ }^{11}$ where the authors proposed to find estimates of difference between luminance and chrominance channels as the result of denoising procedure. In the paper ${ }^{11}$ the concept of directional "demosaicing noise" was introduced for the interpolation errors. A filtering procedure is exploited to remove this noise. In this paper, in order to remove the interpolation errors we exploit the spatially-adaptive LPA-ICI denoising technique $\mathrm{e}^{32,33,34}$ instead of the fix-length filter used in ${ }^{11}$. The ICI rule applied for selection adaptively varying scales (window sizes) of LPA. The LPA-ICI algorithm is nonlinear and spatially-adaptive with respect to the smoothness and irregularities of the image. The efficiency of introducing this step is shown by simulation results.

In many applications the observed data is noisy. We refer reader to ${ }^{35}$ where similar idea is exploited to design a technique that performs both denoising and interpolation of noisy Bayer data. 
The structure of the paper is the following. In Section 2 we introduce the considered Bayer pattern image formation model. The initialization step of the proposed algorithm is described in Section 3. The LPA-ICI filtering is presented in Section 4. In Sections 5-7 the interpolation algorithm of color channels is given. The simulation results are shown in Section 8.

\section{IMAGE FORMATION MODEL}

We follow the general Bayer mask image formation model:

$$
z_{\text {Bayer }}(i, j)=\Psi\left\{y_{R G B}(i, j)\right\},
$$

where $\Psi\{\cdot\}$ is a Bayer sampling operator ${ }^{1}$

$$
\Psi\left\{y_{R G B}(i, j)\right\}=\left\{\begin{array}{l}
G(i, j), \text { at }(i, j) \in X_{G_{1}}, \\
G(i, j), \text { at }(i, j) \in X_{G_{2}}, \\
R(i, j), \text { at }(i, j) \in X_{R} \\
B(i, j), \text { at }(i, j) \in X_{B}
\end{array}\right.
$$

and $z_{\text {Bayer }}$ is an output signal of the sensor, $y_{R G B}(i, j)=(R(i, j), G(i, j), B(i, j))$ is a true color RGB observation scene, $X=\{(i, j): i=1, \ldots, 2 N, j=1, \ldots, 2 M\}$ are the spatial coordinates and $R$ (red), $G$ (green), and $B$ (blue) correspond to the color channels. For two available green channels we will use notations $G_{1}(i, j)$, such that $(i, j) \in X_{G_{1}}=\{(i, j): i=1,3, \ldots, 2 N-1, j=1,3, \ldots, 2 M-1\}$, and $G_{2}(i, j)$, such that $(i, j) \in$ $X_{G_{2}}=\{(i, j): i=2,4, \ldots, 2 N, j=2,4, \ldots, 2 M\}$. Spatial coordinates for the red $R(i, j)$ and blue $B(i, j)$ color channels are denoted $X_{R}=\{(i, j): i=1,3, \ldots, 2 N-1, j=2,4, \ldots, 2 M\}$ and $X_{B}=\{(i, j): i=2,4, \ldots, 2 N$, $j=1,3, \ldots, 2 M-1\}$, respectively.

Demosaicing attempts to invert $\Psi\{\cdot\}$ in order to reconstruct $R(i, j), G(i, j)$, and $B(i, j)$ intensities from the observations $z_{\text {Bayer }}(i, j)$.

The algorithm consists of the following steps: initialization, filtering, and interpolation. At the initialization, the approximate color estimates are obtained and directional differences between $G-R$ and $G-B$ are calculated. These differences are considered as degraded by noise and filtered. The modified LPA-ICI algorithm ${ }^{32,33,34}$ is used for this filtering. Finally, the obtained estimates are exploited to calculate missing color values at each pixel.

\section{INITIALIZATION}

Firstly we calculate the directional (horizontal and vertical) estimates of green channel at every point $(i, j) \in X$ following the rules of Hamilton-Adams algorithm ${ }^{5}$. Interpolation of $G$ at $R$ positions $(i, j) \in X_{R}$ is done as follows:

$$
\begin{aligned}
\hat{G}_{h}(i, j) & =\frac{1}{2}(G(i+1, j)+G(i-1, j))+\frac{1}{4}(-R(i-2, j)+2 R(i, j)-R(i+2, j)), \\
\hat{G}_{v}(i, j) & =\frac{1}{2}(G(i, j+1)+G(i, j-1))+\frac{1}{4}(-R(i, j-2)+2 R(i, j)-R(i, j+2)) .
\end{aligned}
$$

Here $h$ and $v$ stay for horizontal and vertical estimates. Similarly to (3)-(4), the initial directional estimates for the red channel $R$ at green positions $G\left((i, j) \in X_{G_{1}}\right.$ or $\left.(i, j) \in X_{G_{2}}\right)$ are interpolated as:

$$
\begin{aligned}
& \hat{R}_{h}(i, j)=\frac{1}{2}(R(i+1, j)+R(i-1, j))+\frac{1}{4}(-G(i-2, j)+2 G(i, j)-G(i+2, j)), \\
& \hat{R}_{v}(i, j)=\frac{1}{2}(R(i, j+1)+R(i, j-1))+\frac{1}{4}(-G(i, j-2)+2 G(i, j)-G(i, j+2)) .
\end{aligned}
$$


As a result we obtain at the every horizontal line of $R$ values two sets of true and estimated green and red values:

$$
\begin{array}{ccccccc}
\ldots & \hat{G}_{h} & G & \hat{G}_{h} & G & \hat{G}_{h} & \ldots \\
\ldots & R & \hat{R}_{h} & R & \hat{R}_{h} & R & \ldots
\end{array} .
$$

Similar calculations are produced for the vertical lines. At every point the differences between the true values $R(i, j)$ and $G(i, j)$, and the directional estimates $\hat{R}_{h}(i, j)$ and $\hat{G}_{h}(i, j)$, are calculates as follows:

$$
\tilde{\Delta}_{g, r}^{h}(i, j)=G(i, j)-\hat{R}_{h}(i, j),(i, j) \in X_{G_{1}},
$$

and

$$
\tilde{\Delta}_{g, r}^{h}(i, j)=\hat{G}_{h}(i, j)-R(i, j),(i, j) \in X_{R},
$$

for the horizontal direction. For the vertical direction the analogous computations are:

$$
\tilde{\Delta}_{g, r}^{v}(i, j)=G(i, j)-\hat{R}_{v}(i, j),(i, j) \in X_{G_{2}},
$$

and

$$
\tilde{\Delta}_{g, r}^{v}(i, j)=\hat{G}_{v}(i, j)-R(i, j),(i, j) \in X_{R}
$$

We assume for further filtering that these differences between the intensities of different color channels can be presented as the sums of the true values and the random errors:

$$
\begin{aligned}
& \tilde{\Delta}_{g, r}^{h}(i, j)=\Delta_{g, r}(i, j)+\varepsilon_{h}(i, j), \\
& \tilde{\Delta}_{g, r}^{v}(i, j)=\Delta_{g, r}(i, j)+\varepsilon_{v}(i, j),
\end{aligned}
$$

where $\varepsilon_{h}(i, j)$ and $\varepsilon_{v}(i, j)$ are considered as random demosaicing noise ${ }^{11} ; \Delta_{g, r}(i, j)$ is the true difference between green and red color channels.

The blue channel $B$ is treated in the same way and as a result we calculate the directional differences $\tilde{\Delta}_{g, b}^{h}(i, j)$ and $\tilde{\Delta}_{g, b}^{v}(i, j)$.

\section{LPA-ICI FILTERING OF DIRECTIONAL DIFFERENCES}

The LPA-ICI filtering 33,34 is used for all noisy estimates $\tilde{\Delta}_{g, r}^{h}(i, j), \tilde{\Delta}_{g, r}^{v}(i, j)$ for $R$, and $\tilde{\Delta}_{g, b}^{h}(i, j), \tilde{\Delta}_{g, b}^{v}(i, j)$ for $B$. In order to introduce this filtering in the form applicable for any input data assume for a moment that this input noisy data have the form:

$$
z(i, j)=y(i, j)+n(i, j)
$$

where $(i, j) \in X, z(i, j)$ is a noisy observation, $y(i, j)$ is a true signal and $n(i, j)$ is a noise.

The LPA is a general tool for linear filter design, in particular for design of the directional filters of the given orders on the arguments $i$ and $j$. Let $g_{s, \theta}$ be the impulse response of the $2 \mathrm{D}$ directional linear filter designed by the $\mathrm{LPA}^{34}$, where $\theta$ is a direction of smoothing and $s$ is a scale parameter (window size of the filter)*. A set of the image estimates of different scales $s$ and different directions $\theta$ are calculated by the convolution

$$
\widehat{y}_{s, \theta}(i, j)=\left(z \circledast g_{s, \theta}\right)(i, j),
$$

for $s \in S=\left\{s_{1}, s_{2}, \ldots, s_{J}\right\}$, where $s_{1}<s_{2}<\ldots<s_{J}$, and $\theta \in \Theta$.

The ICI rule is the algorithm for a proper selection of the scale (close to the optimal value) for every pixel $(i, j)^{34}$. In the ICI rule a sequence of confidence intervals is used

$$
D_{s}=\left[\widehat{y}_{s, \theta}(i, j)-\Gamma \sigma_{\hat{y}_{s, \theta}}, \widehat{y}_{s, \theta}(i, j)+\Gamma \sigma_{\hat{y}_{s, \theta}}\right], s \in S,
$$

${ }^{*}$ The MATLAB code that implements the LPA-ICI techique is available following the link: http://www.cs.tut.fi/ $\sim$ lasip/. 
where $\Gamma>0$ is a threshold parameter for the ICI, the estimates and $\sigma_{\hat{y}_{s, \theta}}$ is the standard deviation of the estimate $\widehat{y}_{s, \theta}$.

In this paper this standard deviation for (11) is calculated as the weighted mean of the squared errors between the estimates and the observations in the directional neighborhood of the pixel $(i, j)$ :

$$
\sigma_{\hat{y}_{s, \theta}}(i, j)=\sqrt{\left(\left(z-\hat{y}_{s, \theta}\right)^{2} \circledast g_{s, \theta}^{2}\right)(i, j)},
$$

where the weights are defined by $g_{s, \theta}$ used in (10). The rotated directional nonsymmetric kernel $g_{s, \theta}$ is used with the angle $\theta$ which defines the directionality of the filter, and scale $s$ is a length of the kernel support (or a scale parameter of the kernel) in this direction.

Note that in the usual form of the $\operatorname{ICI}^{34}$ the standard deviation of the estimate is calculated as $\sigma_{\hat{y}_{s, \theta}}(i, j)=$ $\sqrt{\left(\sigma^{2} \circledast g_{s, \theta}^{2}\right)(i, j)}$, where $\sigma$ is a given standard deviation of the additive signal-independent observation noise in the model (9). However, in the considered model (7)-(8) we deal with the data where the noise is a convenient form for modelling of the interpolation errors that are actually nonrandom. Thus, the standard deviation of the estimate $\widehat{y}_{s, \theta}$ is estimated locally by (12) at every position $(i, j)$ as an empirical sample mean calculated over the directional local area.

The ICI rule defines the adaptive scale as the largest $s^{+}$of those scales in $S$ which estimate does not differ significantly from the estimates corresponding to the smaller window sizes. This optimization of $s$ for each of the directional estimates yields the adaptive scales $s^{+}(\theta)$ for each direction $\theta$. The union of the supports of $g_{s^{+}}(\theta), \theta$ is considered as an approximation of the best local vicinity of $(i, j)$ in which the estimation model fits the data. The final estimate is calculated as a linear combination of the obtained adaptive directional estimates $\widehat{y}_{s^{+}, \theta}(i, j)$.

The final LPA-ICI estimate $\hat{y}(i, j)$ combined from the directional ones is computed as the weighted mean

$$
\hat{y}(i, j)=\sum_{\theta \in \Theta} \hat{y}_{s^{+}, \theta}(i, j) w_{\theta}, w_{\theta}=\frac{\sigma_{\hat{y}_{s+, \theta}}^{-2}}{\sum_{\theta \in \Theta} \sigma_{\hat{y}_{s}+, \theta}^{-2}}
$$

with the variance $\sigma_{\hat{y}}^{2}$ of $\hat{y}(i, j)$ computed for simplicity as $\sigma_{\hat{y}}^{2}=\left(\sum_{\theta \in \Theta} \sigma_{\hat{y}_{s}+(\theta), \theta}^{-2}\right)^{-1}$.

It is convenient to treat this complex LPA-ICI multidirectional algorithm as an adaptive filter with the input $z$ and the output $\hat{y}$. The input-output equation can be written as $\hat{y}=\mathcal{L} \mathcal{I}\{z\}$ by denoting the calculations imbedded in this algorithm as an $\mathcal{L I}$ operator.

Applying the ICI in the form (11), (12) to $\tilde{\Delta}_{g, r}^{h}(i, j)$ and $\tilde{\Delta}_{g, r}^{v}(i, j)$ we obtain the corresponding spatially adaptive estimates. Let us denote these estimates as $\hat{\Delta}_{g, r}^{h}(i, j)=\mathcal{L I}\left\{\tilde{\Delta}_{g, r}^{h}(i, j)\right\}$ and $\hat{\Delta}_{g, r}^{v}(i, j)=\mathcal{L} \mathcal{I}\left\{\tilde{\Delta}_{g, r}^{v}(i, j)\right\}$.

Combining these vertical and horizontal estimates we arrive to the final estimate $\hat{\Delta}_{g, r}(i, j)$ in the form

$$
\hat{\Delta}_{g, r}(i, j)=\frac{\sigma_{\hat{\Delta}_{g, r}^{h}}^{-2}}{\sigma_{\hat{\Delta}_{g, r}^{h}}^{-2}+\sigma_{\hat{\Delta}_{g, r}^{v}}^{-2}} \hat{\Delta}_{g, r}^{h}(i, j)+\frac{\sigma_{\hat{\Delta}_{g, r}^{v}}^{-2}}{\sigma_{\hat{\Delta}_{g, r}^{h}}^{-2}+\sigma_{\hat{\Delta}_{g, r}^{v}}^{-2}} \hat{\Delta}_{g, r}^{v}(i, j),
$$

where $\sigma_{\hat{\Delta}_{g, r}^{h}}$ and $\sigma_{\hat{\Delta}_{g, r}^{v}}$ are the corresponding standard deviations of $\hat{\Delta}_{g, r}^{h}$ and $\hat{\Delta}_{g, r}^{v}$.

Note that in order to obtain the estimates for $\hat{\Delta}_{q, r}^{h}(i, j)$ we use only two directions corresponding to the directions of interpolation $\Theta=\{0, \pi\}$ (for horizontal left and right estimates), $S=\{4,6,8,12\}$. For vertical estimates $\hat{\Delta}_{g, r}^{v}(i, j)$ we also use two directions corresponding to the directions of interpolation $\Theta=\{\pi / 2,3 \pi / 2\}$ (for vertical up and down estimates).

Similar adaptive LPA-ICI filtering is applied for the differences $\tilde{\Delta}_{g, b}^{h}(i, j), \tilde{\Delta}_{g, b}^{v}(i, j)$ between $G$ and $B$ color channels in order to obtain the estimate $\hat{\Delta}_{g, b}(i, j)$. 

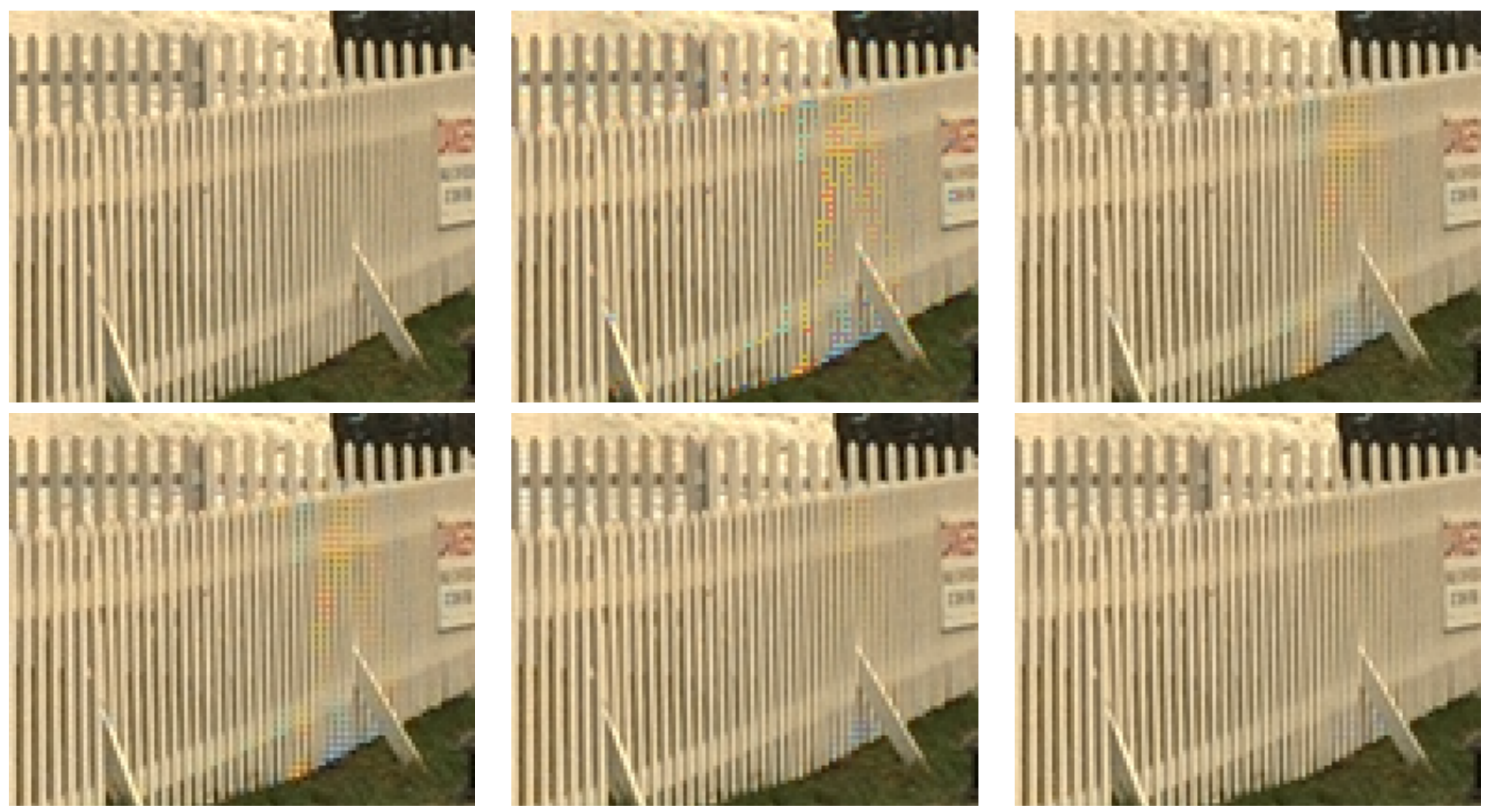

Figure 2. Fragment of the Lighthouse (19) test image (from left to right and from top to bottom): True image; HamiltonAdams interpolation ${ }^{5}$ PSNR=(36.67 38.34 37.09); Successive Approximation ${ }^{8}$ PSNR=(38.67 42.07 40.17); Alternating Projections $^{2}$ PSNR $=\left(38.7042 .12\right.$ 39.80); DLMMSE based interpolation ${ }^{11}$ PSNR $=(39.83$ 42.77 40.93); Proposed LPA-ICI based interpolation PSNR=(40.34 43.5141 .56$)$.

\section{INTERPOLATION OF $G$ COLOR}

The interpolated green color at $R\left((i, j) \in X_{R}\right)$ or $B\left((i, j) \in X_{B}\right)$ positions is calculated as follows:

$$
\begin{aligned}
& \hat{G}(i, j)=R(i, j)+\hat{\Delta}_{g, r}(i, j),(i, j) \in X_{R}, \\
& \hat{G}(i, j)=B(i, j)+\hat{\Delta}_{g, b}(i, j),(i, j) \in X_{B},
\end{aligned}
$$

where $\hat{\Delta}_{g, r}$ is the estimate of $G-R$, and $\hat{\Delta}_{g, b}$ is the estimate of $G-B$, obtained using the LPA-ICI technique (11)-(14) described in the previous section.

\section{INTERPOLATION OF $R / B$ COLORS AT $B / R$ POSITIONS}

For the interpolation of $R / B$ colors at $B / R$ positions we propose to use a special shift invariant interpolation filter giving the estimates by the standard convolution. This filter has been designed using the LPA for the subsampled grid which corresponds to $R / B$ channel (Fig.1). A variety of polynomial orders and support sizes have been tested. Finally, the second order polynomial interpolation filter $g_{r b}$ has been chosen

$$
g_{r b}=\left[\begin{array}{ccccccc}
0 & 0 & -0.0313 & 0 & -0.0313 & 0 & 0 \\
0 & 0 & 0 & 0 & 0 & 0 & 0 \\
-0.0313 & 0 & 0.3125 & 0 & 0.3125 & 0 & -0.0313 \\
0 & 0 & 0 & 0 & 0 & 0 & 0 \\
-0.0313 & 0 & 0.3125 & 0 & 0.3125 & 0 & -0.0313 \\
0 & 0 & 0 & 0 & 0 & 0 & 0 \\
0 & 0 & -0.0313 & 0 & -0.0313 & 0 & 0
\end{array}\right] .
$$




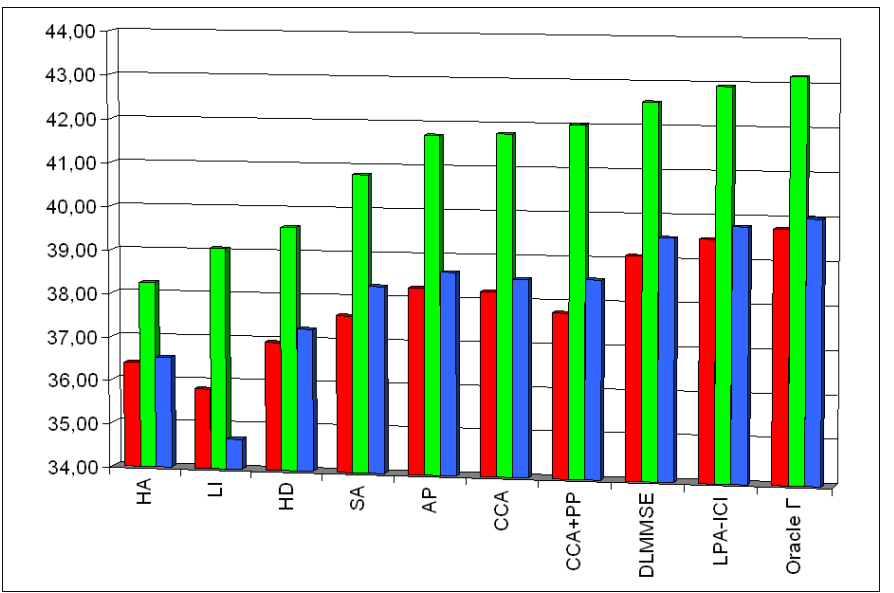

Figure 3. Mean values of PNSR for the Kodak test set of 24 images. The following techniques are compared: $\mathrm{HA}^{5}, \mathrm{LI}^{9}$, $\mathrm{SA}^{8}, \mathrm{HD}^{10}, \mathrm{AP}^{2}, \mathrm{CCA}^{15}, \mathrm{CCA}+\mathrm{PP}$ is a demosaicing approach ${ }^{15}$ with postprocessing ${ }^{7}$, DLMMSE based interpolation ${ }^{11}$, proposed LPA-ICI interpolation, "Oracle $\Gamma$ " is the LPA-ICI interpolation with the optimal threshold parameter $\Gamma$.

Then, the interpolated estimates are computed as follows:

$$
\begin{aligned}
& \hat{R}(i, j)=\hat{G}(i, j)-\left(\hat{\Delta}_{g, r} \circledast g_{r b}\right)(i, j), \quad(i, j) \in X_{B}, \\
& \hat{B}(i, j)=\hat{G}(i, j)-\left(\hat{\Delta}_{g, b} \circledast g_{r b}\right)(i, j), \quad(i, j) \in X_{R},
\end{aligned}
$$

where $\hat{\Delta}_{g, r}$ is the estimate of $G-R$, and $\hat{\Delta}_{g, b}$ is the estimate of $G-B$.

\section{INTERPOLATION OF $R / B$ COLORS AT $G$ POSITIONS}

For interpolation of $R / B$ colors at $G$ positions $\left((i, j) \in X_{G_{1}} \cup X_{G_{2}}\right)$ we use the simplest first order interpolation kernel $g$ :

$$
g=\left[\begin{array}{ccc}
0 & 0.25 & 0 \\
0.25 & 0 & 0.25 \\
0 & 0.25 & 0
\end{array}\right]
$$

because the higher order interpolation does not provide significant improvement in performance. Then, the interpolated estimates are computed as follows:

$$
\begin{aligned}
& \hat{R}(i, j)=G(i, j)-\left(\left(\hat{\Delta}_{g, r} \circledast g_{r b}\right) \circledast g\right)(i, j), \\
& \hat{B}(i, j)=G(i, j)-\left(\left(\hat{\Delta}_{g, b} \circledast g_{r b}\right) \circledast g\right)(i, j) .
\end{aligned}
$$

\section{RESULTS}

The proposed LPA-ICI based CFAI is tested on the Kodak set of color test-images. The numerical results are summarized in Tables 1,2 for each of 24 images and ordered in the ascending order of mean PSNR values (the last row). The diagram that illustrates mean values of PSNR for each color channel is shown in Fig. 3 for the better visual perception. The PSNR criterion is calculated excluding 15 border pixels in order to eliminate boundary effects.

The threshold $\Gamma$ in (11) is an important design parameter of the ICI rule and of the algorithm overall. When $\Gamma$ is small the ICI selects only the estimates with the smallest scale $s$, while when $\Gamma$ is large the ICI selects only the estimates with the largest scale $s$. The best selection of $\Gamma$ for each image can be found if the image is known. We call these values of $\Gamma$ "Oracles". They show the potential of the developed adaptive algorithm provided the best selection of $\Gamma$. The corresponding PSNR values are given in the column "Oracle $\Gamma$ " of Tables 1,2. It is clearly seen that these oracle results are significantly better then results for all other methods. 

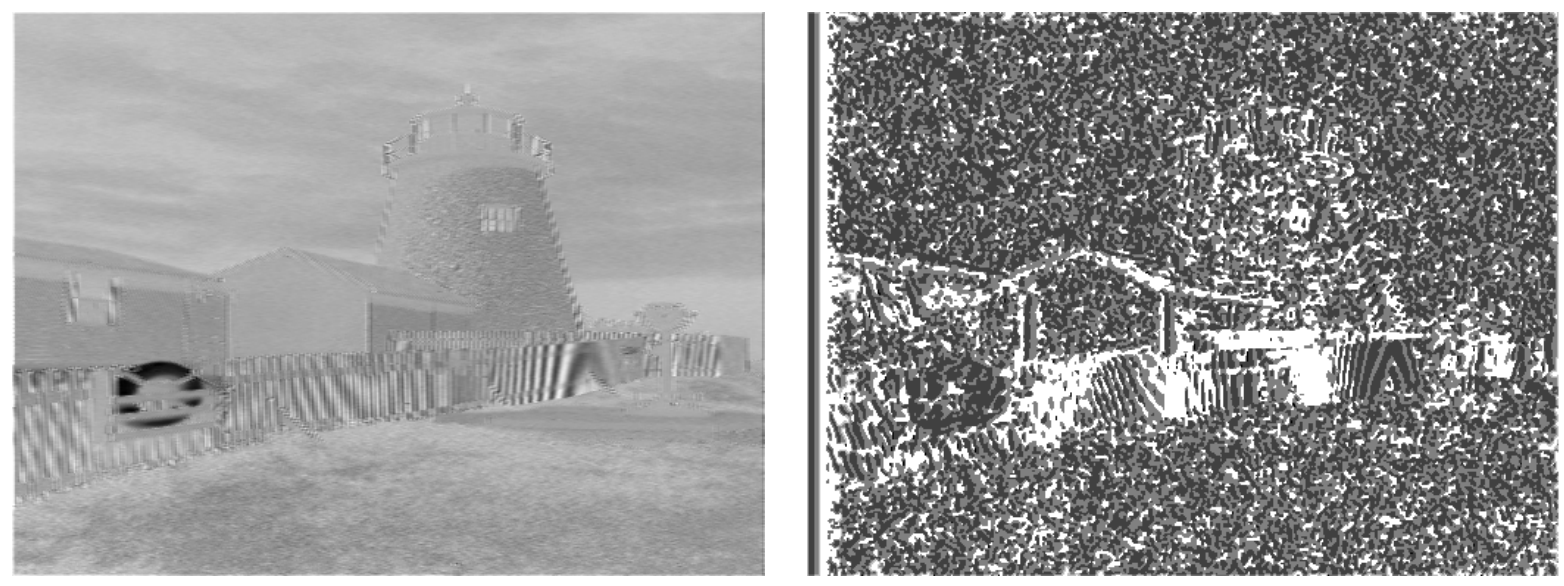

Figure 4. Difference between initial horizontal $G$ and $R$ estimates $(G-R)$ (left) and LPA-ICI scales $(\theta=0)$ of the filtered $(G-R)$ (right).

We found an empirical formula giving the image dependent $\Gamma$ with the values close to the oracle ones. Let $\sigma_{f}$ be standard deviation of high frequency components of $G$ channel calculated as Median Absolute Deviation (MAD). ${ }^{36}$ Then nearly oracle values of the threshold parameter can be calculated as $\Gamma=0.05 \sigma_{f}+0.33$. The results with this value of $\Gamma$ are given in the "LPA-ICI" column of Tables 1,2.

It is clearly seen that the proposed technique ("LPA-ICI" column) gives about $0.4 \mathrm{~dB}$ better mean PSNR value (the last row of Table 2) than DLMMSE ${ }^{11}$ that shows the best performance among the reviewed CFAI methods. Analyzing the diagram in Fig. 3 it can be seen that this improvement is significant.

Fig. 4a illustrates the differences $\tilde{\Delta}_{g, r}^{h}$ between horizontal estimates of green and red color channels obtained for Lighthouse test-image (image number 19 in Table 2). The adaptive scales $s$ for this image (direction $\theta=0$ ) are shown in Fig. 4b. It is seen that near vertical details the filtering performed horizontally selects smaller scales allowing to avoid oversmoothing of details. Our study shows that the "demosaicing noise" is not white and strongly localized. At different parts of an image the power of noise is different. It justifies the use of the local estimates of the variance in (12). As a result, suppression of color distortions becomes much better in terms of both numerical and visual evaluation.

Visual comparison of different methods is presented in Fig. 2 for the fragment of Lighthouse image. The color artefacts are removed almost completely by the proposed method (Fig. 2 bottom right image) what is significantly better than it is done by other methods.

\section{ACKNOWLEDGMENTS}

This work was supported by the Finnish Funding Agency for Technology and Innovation (Tekes). The authors thank Dr. Lei Zhang for providing the implementation code of the technique ${ }^{11}$, and Dr. Alessandro Foi for useful and practical discussions.

\section{REFERENCES}

1. B.E. Bayer, "Color imaging array," U.S. Patent 3971 065, July 1976.

2. B.K. Gunturk, Y. Altunbasak, R.M. Mersereau, "Color plane interpolation using alternating projections", IEEE Transactions on Image Processing, Volume 11, Issue 9, Page(s):997 - 1013, Sept. 2002.

3. J.E. Adams Jr., "Design of color filter array interpolation algorithms for digital cameras, Part 2," in IEEE Proc. Int. Conf. Image Processing, vol. 1, Oct. 1998, pp. 488-492.

4. C.A. Laroche and M.A. Prescott, "Apparatus and method for adaptively interpolating a full color image utilizing chrominance gradients", U.S. Patent 5373 322, Dec. 1994. 


\begin{tabular}{|c|c|c|c|c|c|c|c|c|c|c|c|c|}
\hline & & & $\mathrm{HA}$ & LI & $\mathrm{HD}$ & $\mathrm{SA}$ & AP & $\mathrm{CCA}$ & $\mathrm{CCA}+\mathrm{PP}$ & DLMMSE & LPA-ICI & Oracle $\Gamma$ \\
\hline \multirow{3}{*}{01} & & Red & 33.17 & 30.87 & 34.51 & 36.99 & 36.69 & 36.22 & 37.25 & 37.58 & 39.65 & 39.88 \\
\hline & & Green & 34.65 & 35.61 & 36.13 & 40.76 & 40.42 & 39.20 & 41.31 & 40.22 & 42.80 & 43.08 \\
\hline & & Blue & 33.29 & 30.98 & 34.74 & 38.77 & 37.26 & 36.72 & 38.29 & 38.02 & 40.15 & 40.48 \\
\hline \multirow{3}{*}{02} & & Red & 37.38 & 36.54 & 36.83 & 35.50 & 37.29 & 37.76 & 36.52 & 38.19 & 38.59 & 38.72 \\
\hline & & Green & 40.94 & 41.35 & 41.61 & 40.57 & 42.46 & 43.81 & 43.27 & 44.31 & 44.59 & 44.64 \\
\hline & & Blue & 39.60 & 37.32 & 40.44 & 40.05 & 40.84 & 41.35 & 41.06 & 42.54 & 42.78 & 42.81 \\
\hline \multirow{3}{*}{03} & & Red & 40.21 & 39.22 & 40.39 & 39.19 & 40.94 & 41.58 & 39.90 & 41.95 & 42.92 & 42.91 \\
\hline & & Green & 42.19 & 43.15 & 43.56 & 41.00 & 43.52 & 45.18 & 43.78 & 45.80 & 46.00 & 46.03 \\
\hline & & Blue & 39.79 & 38.37 & 40.03 & 38.84 & 40.34 & 41.20 & 40.30 & 41.40 & 42.29 & 42.30 \\
\hline \multirow{3}{*}{04} & & Red & 36.58 & 36.73 & 35.85 & 35.25 & 36.87 & 37.40 & 35.92 & 37.21 & 37.37 & 37.87 \\
\hline & & Green & 40.66 & 42.32 & 41.47 & 41.63 & 43.81 & 44.55 & 44.26 & 44.68 & 44.09 & 44.55 \\
\hline & & Blue & 39.64 & 38.91 & 40.74 & 41.91 & 42.33 & 42.46 & 42.32 & 43.61 & 43.19 & 43.38 \\
\hline \multirow{3}{*}{05} & & Red & 34.65 & 32.60 & 34.95 & 34.60 & 36.87 & 37.12 & 36.10 & 37.62 & 36.26 & 38.06 \\
\hline & & Green & 35.81 & 36.75 & 37.27 & 36.78 & 39.69 & 40.02 & 39.61 & 40.88 & 39.26 & 41.05 \\
\hline & & Blue & 34.27 & 32.25 & 34.27 & 34.26 & 36.06 & 36.42 & 35.72 & 36.71 & 35.67 & 37.17 \\
\hline \multirow{3}{*}{06} & & Red & 34.66 & 32.49 & 37.35 & 39.02 & 38.22 & 36.86 & 38.12 & 40.13 & 40.90 & 40.93 \\
\hline & & Green & 35.93 & 36.91 & 38.88 & 42.22 & 41.48 & 39.86 & 41.68 & 42.33 & 43.71 & 43.76 \\
\hline & & Blue & 34.24 & 31.98 & 36.49 & 38.00 & 37.35 & 36.37 & 37.43 & 38.80 & 39.33 & 39.33 \\
\hline \multirow{3}{*}{07} & & Red & 40.64 & 38.77 & 39.99 & 39.25 & 41.25 & 41.87 & 40.07 & 41.83 & 42.59 & 42.68 \\
\hline & & Green & 42.17 & 42.16 & 42.64 & 41.22 & 43.96 & 45.31 & 43.97 & 45.27 & 45.48 & 45.52 \\
\hline & & Blue & 40.25 & 38.51 & 39.37 & 38.75 & 40.69 & 41.32 & 39.91 & 41.01 & 41.74 & 41.86 \\
\hline \multirow{3}{*}{08} & & Red & 31.57 & 28.05 & 33.06 & 34.55 & 34.56 & 33.34 & 34.44 & 35.08 & 36.12 & 36.08 \\
\hline & & Green & 33.37 & 32.96 & 35.19 & 38.45 & 38.55 & 36.93 & 38.63 & 38.53 & 40.07 & 40.07 \\
\hline & & Blue & 31.55 & 27.83 & 33.11 & 35.28 & 34.67 & 33.46 & 34.82 & 35.23 & 36.34 & 36.32 \\
\hline \multirow{3}{*}{09} & & Red & 39.38 & 36.78 & 40.12 & 39.72 & 40.64 & 40.99 & 40.29 & 41.69 & 42.27 & 42.30 \\
\hline & & Green & 41.39 & 41.40 & 42.83 & 42.02 & 43.42 & 43.94 & 43.90 & 45.14 & 45.36 & 45.42 \\
\hline & & Blue & 40.23 & 37.39 & 40.45 & 40.90 & 41.90 & 40.92 & 40.91 & 43.00 & 43.22 & 43.31 \\
\hline \multirow{3}{*}{10} & & Red & 39.12 & 37.64 & 39.43 & 40.25 & 40.78 & 40.83 & 39.94 & 41.19 & 41.45 & 41.76 \\
\hline & & Green & 41.33 & 42.27 & 42.80 & 43.91 & 44.38 & 44.42 & 44.41 & 45.37 & 45.34 & 45.53 \\
\hline & & Blue & 39.49 & 37.69 & 39.89 & 41.45 & 41.41 & 40.96 & 40.67 & 42.08 & 41.99 & 42.19 \\
\hline \multirow{3}{*}{11} & & Red & 35.50 & 33.71 & 36.44 & 37.43 & 37.96 & 37.71 & 37.69 & 38.75 & 39.03 & 38.97 \\
\hline & & Green & 37.03 & 37.90 & 38.76 & 41.20 & 41.64 & 41.25 & 42.15 & 42.03 & 42.81 & 42.81 \\
\hline & & Blue & 35.87 & 33.65 & 37.25 & 39.03 & 38.92 & 38.70 & 39.32 & 39.87 & 40.19 & 40.16 \\
\hline \multirow{3}{*}{12} & & Red & 39.89 & 37.44 & 40.45 & 40.89 & 41.41 & 40.97 & 40.45 & 42.09 & 42.82 & 42.87 \\
\hline & (1) & Green & 42.27 & 42.41 & 43.82 & 44.38 & 45.22 & 44.74 & 44.82 & 46.30 & 46.62 & 46.64 \\
\hline & & Blue & 40.21 & 37.74 & 41.11 & 42.21 & 42.09 & 41.31 & 41.46 & 42.98 & 43.38 & 43.41 \\
\hline$\ldots$ & $\ldots$ & $\ldots$ & $\ldots$ & $\ldots$ & $\ldots$ & $\ldots$ & $\ldots$ & $\ldots$ & $\ldots$ & $\ldots$ & $\ldots$ & $\ldots$ \\
\hline
\end{tabular}

Table 1. PSNR comparison for different demosaicing methods computed within 15 pixels border: $\mathrm{HA}^{5}, \mathrm{LI}^{9}, \mathrm{HD}^{10}, \mathrm{SA}^{8}$, $\mathrm{AP}^{2}, \mathrm{CCA}^{15}, \mathrm{CCA}+\mathrm{PP}$ is a demosaicing approach ${ }^{15}$ with postprocessing ${ }^{7}$, DLMMSE based interpolation ${ }^{11}$, proposed LPA-ICI based interpolationin, "Oracle $\Gamma$ " is the LPA-ICI based interpolation with the optimal threshold parameter $\Gamma$.

5. J.F. Hamilton Jr. and J.E. Adams, "Adaptive Color plane Interpolation in single color electronic camera", U.S. Patent 5629 734, May 1997.

6. R. Kimmel, "Demosaicing: image reconstruction from color CCD samples", IEEE Transactions on Image Processing, Volume 8, Issue 9, Page(s):1221 - 1228, Sept. 1999.

7. R. Lukac, Martin K., Plataniotis K.N., "Demosaicked Image Postprocessing Using Local Color Ratios", IEEE Transactions on Circuits and Systems for Video Technology, Vol. 14, No. 6, pp. 914-920, June 2004.

8. Xin Li, "Demosaicing by successive approximation", IEEE Transactions on Image Processing, Volume 14, Issue 3, Page(s):370 - 379, March 2005. 


\begin{tabular}{|c|c|c|c|c|c|c|c|c|c|c|c|c|}
\hline & & & $\mathrm{HA}$ & LI & $\mathrm{HD}$ & $\mathrm{SA}$ & $\mathrm{AP}$ & $\mathrm{CCA}$ & $\mathrm{CCA}+\mathrm{PP}$ & DLMMSE & LPA-ICI & Oracle $\Gamma$ \\
\hline \multirow{3}{*}{13} & & Red & 29.53 & 28.86 & 31.32 & 36.00 & 34.04 & 34.07 & 35.84 & 34.98 & 36.39 & 36.48 \\
\hline & & Green & 30.58 & 32.53 & 32.18 & 38.38 & 36.83 & 36.02 & 38.13 & 36.09 & 38.13 & 38.14 \\
\hline & & Blue & 29.10 & 28.36 & 30.43 & 34.05 & 32.86 & 32.97 & 34.00 & 33.56 & 34.27 & 34.37 \\
\hline \multirow{3}{*}{14} & & Red & 34.81 & 33.74 & 33.82 & 31.59 & 34.57 & 35.68 & 33.77 & 35.53 & 34.87 & 36.53 \\
\hline & & Green & 37.14 & 37.72 & 37.73 & 34.98 & 38.04 & 40.25 & 39.00 & 40.28 & 38.98 & 40.55 \\
\hline & & Blue & 35.02 & 33.31 & 34.62 & 32.64 & 35.11 & 36.42 & 35.11 & 36.25 & 35.70 & 37.03 \\
\hline \multirow{3}{*}{15} & & Red & 36.08 & 36.50 & 35.69 & 35.68 & 36.79 & 36.95 & 36.10 & 37.22 & 37.55 & 37.74 \\
\hline & & Green & 39.51 & 41.56 & 40.62 & 40.59 & 42.29 & 42.79 & 42.32 & 43.23 & 42.94 & 43.13 \\
\hline & & Blue & 37.96 & 37.57 & 38.82 & 39.78 & 40.22 & 40.59 & 40.62 & 41.24 & 41.10 & 41.18 \\
\hline \multirow{3}{*}{16} & & Red & 38.09 & 35.44 & 41.20 & 42.11 & 41.56 & 39.73 & 41.14 & 43.60 & 44.06 & 44.23 \\
\hline & & Green & 39.55 & 40.01 & 42.66 & 45.46 & 44.82 & 42.87 & 44.60 & 45.75 & 46.51 & 46.83 \\
\hline & & Blue & 37.88 & 35.30 & 40.41 & 41.08 & 40.85 & 39.49 & 40.49 & 42.49 & 42.86 & 42.84 \\
\hline \multirow{3}{*}{17} & & Red & 38.41 & 36.95 & 38.93 & 40.88 & 40.79 & 40.77 & 40.06 & 41.38 & 41.28 & 41.38 \\
\hline & & Green & 39.19 & 40.38 & 40.36 & 43.17 & 43.03 & 42.94 & 43.22 & 43.15 & 43.40 & 43.42 \\
\hline & & Blue & 37.74 & 36.67 & 38.32 & 40.52 & 40.31 & 39.92 & 39.80 & 40.83 & 40.72 & 40.81 \\
\hline \multirow{3}{*}{18} & & Red & 33.91 & 33.40 & 34.21 & 35.32 & 36.23 & 36.56 & 35.90 & 36.69 & 36.27 & 36.75 \\
\hline & & Green & 35.24 & 36.92 & 36.12 & 38.36 & 39.46 & 39.42 & 39.73 & 39.41 & 39.30 & 39.54 \\
\hline & & Blue & 33.75 & 33.00 & 34.26 & 36.41 & 36.86 & 36.71 & 36.88 & 37.27 & 36.90 & 37.23 \\
\hline \multirow{3}{*}{19} & & Red & 36.67 & 32.61 & 37.60 & 38.67 & 38.70 & 37.90 & 38.41 & 39.83 & 40.34 & 40.32 \\
\hline & & Green & 38.34 & 37.24 & 39.51 & 42.07 & 42.12 & 41.08 & 42.25 & 42.77 & 43.51 & 43.53 \\
\hline & & Blue & 37.09 & 32.64 & 37.86 & 40.17 & 39.80 & 38.16 & 38.85 & 40.93 & 41.56 & 41.57 \\
\hline \multirow{3}{*}{20} & & Red & 38.75 & 36.98 & 39.29 & 40.54 & 40.96 & 41.17 & 40.66 & 41.80 & 41.94 & 41.97 \\
\hline & & Green & 39.82 & 40.57 & 40.85 & 42.79 & 43.50 & 43.76 & 43.87 & 43.86 & 44.10 & 44.27 \\
\hline & & Blue & 37.31 & 35.72 & 37.55 & 38.12 & 38.71 & 39.24 & 38.98 & 39.27 & 39.67 & 39.68 \\
\hline \multirow{3}{*}{21} & & Red & 35.04 & 33.40 & 36.46 & 38.86 & 38.47 & 38.31 & 39.14 & 39.14 & 39.93 & 40.00 \\
\hline & & Green & 36.29 & 37.47 & 37.76 & 41.92 & 41.57 & 40.90 & 42.32 & 41.22 & 42.31 & 42.45 \\
\hline & & Blue & 34.48 & 32.74 & 35.41 & 37.50 & 37.19 & 37.16 & 37.70 & 37.65 & 38.12 & 38.09 \\
\hline \multirow{3}{*}{22} & & Red & 35.80 & 34.90 & 35.41 & 36.53 & 37.03 & 37.22 & 36.06 & 37.60 & 37.41 & 37.73 \\
\hline & & Green & 37.72 & 38.44 & 38.22 & 38.69 & 39.72 & 40.58 & 39.88 & 40.86 & 40.53 & 40.91 \\
\hline & & Blue & 35.60 & 33.84 & 35.48 & 36.18 & 36.71 & 37.13 & 36.55 & 37.42 & 37.27 & 37.67 \\
\hline \multirow{3}{*}{23} & & Red & 41.06 & 39.83 & 40.31 & 38.50 & 40.76 & 41.48 & 39.25 & 41.78 & 42.69 & 42.78 \\
\hline & & Green & 43.30 & 43.71 & 43.92 & 41.44 & 44.03 & 45.60 & 44.03 & 46.24 & 46.28 & 46.36 \\
\hline & & Blue & 41.54 & 40.27 & 41.15 & 39.51 & 41.61 & 42.40 & 41.02 & 42.78 & 43.25 & 43.39 \\
\hline \multirow{3}{*}{24} & & Red & 32.63 & 32.24 & 32.70 & 34.70 & 34.94 & 34.50 & 33.53 & 35.94 & 35.38 & 35.67 \\
\hline & & Green & 33.59 & 35.61 & 35.15 & 37.38 & 37.49 & 37.45 & 37.41 & 38.01 & 38.05 & 38.09 \\
\hline & & Blue & 30.76 & 30.47 & 31.90 & 33.03 & 32.93 & 32.95 & 32.82 & 33.74 & 33.55 & 33.65 \\
\hline \multirow{3}{*}{\multicolumn{2}{|c|}{ Mean PSNR }} & Red & 36.40 & 35.82 & 36.93 & 37.58 & 38.26 & 38.21 & 37.77 & 39.11 & 39.51 & 39.77 \\
\hline & & Green & 38.25 & 39.06 & 39.58 & 40.81 & 41.73 & 41.79 & 42.02 & 42.57 & 42.93 & 43.18 \\
\hline & & Blue & 36.53 & 34.69 & 37.26 & 38.27 & 38.63 & 38.51 & 38.54 & 39.53 & 39.80 & 40.01 \\
\hline
\end{tabular}

Table 2. PSNR comparison for different demosaicing methods computed within 15 pixels border: $\mathrm{HA}^{5}, \mathrm{LI}^{9}, \mathrm{HD}^{10}, \mathrm{SA}^{8}$, $\mathrm{AP}^{2}, \mathrm{CCA}^{15}, \mathrm{CCA}+\mathrm{PP}$ is a demosaicing approach ${ }^{15}$ with postprocessing ${ }^{7}$, DLMMSE based interpolation ${ }^{11}$, proposed LPA-ICI based interpolationin, "Oracle $\Gamma$ " is the LPA-ICI based interpolation with the optimal threshold parameter $\Gamma$.

9. H.S. Malvar, Li-wei He, and R. Cutler, "High-quality linear interpolation for demosaicing of Bayer-patterned color images", IEEE International Conference (ICASSP '04), Proceedings on Acoustics, Speech, and Signal Processing, Volume 3, Page(s):iii - 485-8, 17-21 May 2004.

10. K. Hirakawa, T.W. Parks, "Adaptive homogeneity-directed demosaicing algorithm", IEEE Transactions on Image Processing, Volume 14, Issue 3, Page(s):360 - 369, March 2005.

11. L. Zhang, X. Wu, "Color Demosaicking Via Directional Linear Minimum Mean Square-Error Estimation", 
IEEE Transactions on Image Processing, Vol. 14, No. 12, pp. 2167-2178, December 2005.

12. J.W. Glotzbach, R.W. Schafer, and K. Illgner, "A method of color filter array interpolation with alias cancellation properties," in Proc. Int. Conf. Image Processing, vol. 1, Oct. 2001, pp. 141-144.

13. X. Wu, N. Zhang, "Primary-consistent soft-decision color demosaicking for digital cameras (patent pending)", IEEE Transactions on Image Processing, Volume 13, Issue 9, Page(s):1263 - 1274, Sept. 2004.

14. D.D. Muresan, T.W. Parks, "Demosaicing Using Optimal Recovery", IEEE Transactions on Image Processing, Vol. 14, No. 2, pp. 267-278, February, 2005.

15. R. Lukac, Plataniotis K.N., Hatzinakos D., Aleksic M., "A Novel Cost Effective Demosaicing Approach", IEEE Transactions on Consumer Electronics, Vol. 50, No. 1, pp. 256-261, February 2004.

16. E. Chang, S. Cheung and D. Y. Pan, "Color filter array recovery using a threshold-based variable number of gradients", Proceedings of SPIE, vol. 3650, pp. 36-43, 1999.

17. R. Lukac, K.N. Plataniotis, "Digital Camera Zooming on Colour Filter Array", Electronics Letters, Vol. 39, No. 25, December 2003.

18. Xiaomeng Wang, Weisi Lin, Ping Xue, "Edge-Adaptive Color Reconstruciton for Single-Sensor Digital Cameras", ICICS-PCM 2003 Singapore, pp. 272-276, December, 2003.

19. Wenmiao Lu, Yap-Peng Tan, "Color Filter Array Demosaicking: New Method and Performance Measures", IEEE Transactions on Image Processing, Vol. 12, No. 10, pp. 1194-1210, October, 2003.

20. X. Wu, W. K. Choi, and P. Bao, "Color restoration from digital camera data by pattern matching", Proc. SPIE, vol. 3018, pp. 12-17, 1997.

21. D. Taubman, "Generalized Weiner reconstruction of images from colour sensor data using a scale invariant prior", Proc Int. Conf. Image Proc., pp. 801-804, 2000.

22. S.-C. Pei and I.-K. Tam, "Effective color interpolation in CCD color filter arrays using signal correlation", IEEE Trans. Circuits Systems Video Technol., vol. 13, no. 6, pp. 503-513, Jun. 2003.

23. Gunturk B.K., Glotzbach J., Altunbasak Y., Schafer R., Mersereau R.M., "Demosaicking: Color Filter Array Interpolation", IEEE Signal Processing Magazine, pp. 44-54, January 2005.

24. D. Alleysson, S. Susstrunk, J. Marguier, "Linear Demosaicing Inspired by the Human Visual System", IEEE Transactions on Image Procesing, Vol. 14, No. 4, April 2005.

25. D. Alleysson, S. Susstrunk, J. Herault, "Color demosaicing by estimating luminance and opponent chromatic signals in the Fourier domain", in Proc. IS\&T/SID 10th Color Imaging Conf., pp. 331-336, Nov. 2002.

26. D. R. Cok, "Reconstruction of CCD Images Using Template Matching", IS\&T's 47th Annual Conference/ICPS, pp380-385, 1994.

27. H.J. Trussel, E. Saber, M. Vrhel, "Color Image Processing: Vector Filtering for Color Imaging", IEEE Signal Processing Magazine, pp. 14-22, January 2005.

28. D. Keren, M. Osadchy, "Restoring Subsampled Color Images", Machine Vision and Applications, pp. 197202, 1999.

29. D.H. Brainard, "Bayesian method for reconstructing color images from trichromatic samples", IS $\backslash \&$ T's 47th Annual Conference/ICSP, pp 375-380, 1994.

30. R. Lukac, K.N. Plataniotis, "An Efficient CFA Interpolation Solution, 46th International Symposium Electronics in Marine", ELMAR-2004, Zadar, Croatioa, June, 2004.

31. R. Lukac, B. Smolka, K. Martin, K.N. Plataniotis, A.N. Venetsanopulos, "Vector Filtering for Color Imaging", IEEE Signal Processing Magazine, pp. 74-86, January 2005.

32. V. Katkovnik, "A new method for varying adaptive bandwidth selection", IEEE Trans. on Signal Proc., vol. 47 , no. 9, pp. 2567-2571, 1999.

33. V. Katkovnik, K. Egiazarian, and J. Astola, "Adaptive window size image de-noising based on intersection of confidence intervals (ICI) rule", J. of Math. Imaging and Vision, vol. 16, no. 3, pp. 223-235, 2002.

34. V. Katkovnik, K. Egiazarian, and J. Astola, Local Approximation Techniques in Signal and Image Processing, SPIE Press, Monograph Vol. PM157, Hardcover, 576 pages, ISBN 0-8194-6092-3, September 2006.

35. Paliy D., M. Trimeche, V. Katkovnik, S. Alenius, "Demosaicing of Noisy Data: Spatially Adaptive Approach", Proc. SPIE Electronic Imaging 2007, Computational Imaging IV, 6497-20, San Jose, CA, January 2007.

36. D.L. Donoho, "De-noising by soft-thresholding," IEEE Tans. Inform. Theory, vol. 41, no. 3, pp. 613-627, May 1995. 